\title{
CHESS TRAINING EFFECT ON META-COGNITIVE PROCESSES AND ACADEMIC PERFORMANCE
}

\author{
Carla Meloni and Rachele Fanari \\ Department of Education, Psychology, Philosophy \\ University of Cagliari, Italy
}

\begin{abstract}
The aim of this study is to examine, in an educational context, the influence of chess training on academic performance (written text comprehension and recall and mathematical problem-solving ability) and on meta-cognitive skills (approach to studying and study strategies availability). A sample of 85 children attending primary school participated in the study: 48 children in the experimental group and 37 in the control group. The experimental group took part to a chess training (a 30-hour chess program) during school hours; the control group carried out a sport program. The results show that after the chess training, the two groups did not differ in their approach to studying, in their use of more or less functional study strategies, and in their written text recall and comprehension ability; instead, a significant difference emerged between the two groups in mathematical problem solving: The experimental group children showed a greater ability to represent a math problem and to categorize it than the control children. The results will be discussed in light of the debate about the transfer of specific domain skills to general domain skills.
\end{abstract}

\section{KEYWORDS}

Chess Training, Math Problem, Reading Comprehension, Meta-Cognitive Ability

\section{INTRODUCTION}

In several European countries, projects involving the introduction of chess instruction in primary school are promoted. Many schools offer chess as an optional subject, while for some schools chess teaching is a part of the standard school program; this also happens following the favorable opinion of the European Parliament itself that promotes the chess game as an important educational tool (Binev, Attard-Montalto, Deva, Mauro, \& Takkula, 2011).

The hypothesis behind these choices is that the skills acquired in this specific field can improve academic performance, both in mathematics and reading, and may lead to an improvement even in general domain of cognitive skills. The idea is, therefore, that the specific skills acquired in the chess practice can be transferred to other domains (see Sala \& Gobbet, 2016 for a discussion).

Transfer is a process that occurs when skills acquired in a given domain are transferred to another specific or general domain, but the exact nature of the transfer process is not yet entirely clear.

In 1901, Thorndike and Woodworth formulated the hypothesis that transfer depends on the number of features shared between two domains. More recently, Anderson (1990) stated that transfer is a function of the degree of overlap of the cognitive elements present in two tasks, an idea suggesting that the transfer from one specific task to another is often limited. Sternberg (2000) suggested a different approach to the transfer issue: transferable abilities are those constituting the basis of intelligence (general abilities as the verbal or visuo-spatial abilities) that can be applied in different domains but that, being innate, cannot be increased through practice.

Some experimental evidence (for example, Ericsson \& Charness, 1994) has shown that the higher the level of expertise in a given specific domain, the more the transfer is limited. Generic learning skills (learning strategies, problem-solving methods, and reasoning techniques), on the other hand, are useful for more domains, but their teaching seems to have immediate, but not long-term benefits (Grotzer \& Perkins, 2000). 
Regarding the potential of transferring of skills acquired in chess playing to other domains, Gobet and Campitelli (2005), in a critical review, emphasized that the results of the works done on the topic, even if they seem to support a possible transfer of abilities, are often weak and contradictory due to methodological problems. Based on their review, the empirical evidence suggests that chess players tend to be smarter than non-chess players, and that, at least with children, there is a correlation between chess skills and general intelligence even if, quite surprisingly, a direct link between chess and visuospatial skills has not been identified. However, these results could be explained mainly by sample selection processes: more intelligent people are more likely to choose, and to excel, in intellectual activities such as chess.

In a recent meta-analysis, Sala, Foley, and Gobbet (2016) investigated the effects of chess programs both on cognitive abilities and school performances in primary school children. The authors concluded that the effects of chess training are more evident, even if moderate, on math performance and general cognitive skills than reading skills. At least $25-30$ hours of chess training seem to be required to have positive effects. In the literature, it is often stated that chess playing improves math skills because chess practice has some elements in common with the domain of mathematics and promotes skills independent from the chess-specific context, such as the ability to understand the existence of a problem and reasoning skills.

Only a few studies have investigated the relationship between chess practice and both general meta-cognitive abilities and specific academic school skills (e.g., reading and math abilities) in the same experimental design, but one problem with these studies making it difficult to compare results and to draw clear conclusions is the significant variability of the variable considered (arithmetic vs. problem solving; reading and text comprehension; attention vs spatial abilities or meta-cognitive abilities). To our knowledge, there are only one study that have investigated the effects of chess training on meta-cognitive abilities, focused on metacognitive skills specifically related to math performance. The study, published in 2012 by Kazemi, Yektayar, and Abad found a positive effect of the use of chess programs on math meta-cognitive abilities. The authors concluded that chess instruction is a way to develop higher-order thinking skills useful for math problem solving.

Most research on meta-cognitive abilities and chess training in primary schools concentrates on the transfer of specific chess skills to specific meta-cognitive abilities used in the math domain. Less is known on chess training's influence on general meta-cognitive skills, skills that children can use in other domains, such motivation to study, organization of personal work, strategic elaboration of the learning material, flexibility of the modality of studying, ability to concentrate, anxiety and attitude toward school, and the knowledge and use of more or less functional study strategies.

The aim of our work is to fill this gap and to explore the link between chess training and both general meta-cognitive study abilities and verbal and math academic skills in primary school children.

We compared two groups of children - one group participating in a chess training and a control group - in their ability to solve math problems, to comprehend and recall a written text, and in their approach to studying and using study strategies.

Following the literature on the relationship between chess playing and academic skills, we expect chess training to influence mathematical problem-solving abilities, but not verbal skills. There is no literature on the effect of chess training on general meta-cognitive abilities of the approach to studying and on the knowledge and use of more or less functional strategies of studying. Therefore, the study of these aspects represents a particularly innovative component of our work and can cast light on the debated issue of the potential to improve general meta-cognitive abilities, useful in various domains, through the training of a specific skill.

\section{METHOD}

\subsection{Participants}

Eighty-five typically developing children were recruited from a public primary school in Cagliari, Italy. Both the school and the children's parents agreed to let the children take part in the research study and signed informed consent forms. 
Forty-eight children were randomly assigned to a chess training group (mean age $=9.27$ years and $\mathrm{SD}=0.84 ; 24$ males and 24 females), and 37 were randomly assigned to a control group (mean age $=9.25$ years and $\mathrm{SD}=0.76 ; 17$ males and 20 females). At the start of the study, all the children were chess novices. The participants came from different classes in which the same teachers evenly rotated; thus, any teacher effects were controlled and the teaching provided to the children was the same even if the children came from different classes. After conducting the random assignment to the experimental and control groups, the teachers were asked if they believed, based on their daily experience with the children, that there were differences between the two groups related to academic performance or differences in attitudes toward school/learning. The teachers noted that the two randomly selected groups were comparable with respect to these variables.

\subsection{Procedure}

The children in the experimental group participated in a chess program during the school year. Chess lessons were held by a chess master once per week from November to May during school hours. Following Sala et al.'s (2016) meta-analysis results, a 30-hour program was chosen. The control group followed a sport training: specifically, an introduction to basketball.

At the end of the training, the children were presented with a test battery aimed to assess their meta-cognitive skills (approach to studying, knowledge and actual use of functional and dysfunctional strategies) and were tested on their ability to solve mathematical problems and on their level of text comprehension and text recall.

\subsection{Assessment Tools}

The tools used to assess children's meta-cognitive abilities were taken from the AMOS 8-15 Skills and Motivation Study Battery (De Beni, Moè \& Cornoldi, 2003). The battery is composed of seven questionnaires indicating different aspects of meta-cognitive abilities involved in academic performance. The three questionnaires we used in this work were: the QAS questionnaire measuring the approach to studying, the QS1 questionnaire measuring the effectiveness of the study strategies know by the children, and the QS2 questionnaire evaluating the children's actual use of the study strategies.

The QAS questionnaire on the approach to studying investigates seven different dimensions (part A: study motivation; part B: personal work organization; part D: strategic information processing; part E: study flexibility; part N: concentration; part U: anxiety; part V: attitude toward school), for each dimension, seven different statements are presented to the child and he/she must indicate with a cross how true each written statement is to him/her $(1=$ not true, $2=$ enough true, and $3=$ very true $)$. The QAS allows for a total score for the approach to studying ability as well as a single score for each dimension.

The second questionnaire used, QS1, identifies the children's beliefs on the effectiveness of functional and dysfunctional strategies that can be used while studying. In particular, the QS1 measures 32 studying strategies (example item: "Thinking about what you already known about the topic you are studying"), and the child is asked to read them carefully and evaluate how much these strategies, according to him/her, are useful for studying, giving each strategy a rating from 1 to $4(1=$ not useful, $2=$ not very useful, $3=$ useful, and 4 = very useful). The third questionnaire, QS2, detects the child's actual use of the same strategies proposed by the QS1 questionnaire. In the QS2, therefore, 32 studying strategies are proposed (example item: "If you do not understand a part of the text, read it again") and the child is asked to think about their approach to studying and to indicate how often he/she uses the activity with a rating from 1 to $4(1=$ I never use it, 2 = I use it sometimes, $3=$ I use it often, and $4=$ I always use it). These two last questionnaires (QS1 and QS2) allow a summary index of strategic coherence to be calculated that reflects the correspondence between utility judgments and the estimation of the use of the same strategies by children.

The tools used to assess children's school performance were the "Mathematical Problem Solving" (SPM) test (Lucangeli, Tressoldi \& Cedron, 2003) used to test mathematical problem solving and a test taken from the AMOS 8-15 Battery (De Beni, Moè ,\& Cornoldi, 2003) called the "Studying Test." 
In the SPM, the child is presented with some mathematical problems with different difficulty levels depending on the level of schooling. The SPM evaluates the following skills: problem understanding (understanding the information present in the problem and their relationships), problem representation (the representation of information through a scheme able to integrate problem information), problem categorization (ability to identify among a series of alternatives the problem that has the same deep structure), problem-solving planning, problem-solving procedure, and self-assessment of the correctness of the used procedure.

Moreover, to evaluate the ability to understand, store, and recall information, the Studying Test was used. The test asks the children to study a written text for 30 minutes. After 30 minutes, they are involved in other activities for 10 minutes and then questions about the text are presented to evaluate three indexes: the ability to select the main aspects of the text (asking the child to choose a suitable title), the ability to identify specific information (open questions), and the ability to recognize true/false information with respect to the studied text (multiple-choice test).

\subsection{Results}

For the data analysis, the scores obtained in each test were calculated following the indications provided. Since the participants in the study were of different ages and school classes, the raw scores were transformed into Z points to compare children's scores, following the test norms.

We conducted six multivariate analyses of variance (MANOVAs). All the MANOVAs had the factor "training" as the independent variable with two levels: chess training and control.

The first MANOVA was carried out to analyze the effects of training on the dimensions of the approach to studying investigated by the QAS; the second, third, and fourth MANOVA were carried out to analyze, respectively: the effects of training on children's beliefs on the effectiveness of functional and dysfunctional study strategies (QS1 questionnaire), the effects of training on the actual use of functional and dysfunctional study strategies (QS2 questionnaire), and the index of strategic coherence in the usage of study strategies; the fifth MANOVA was carried out to analyze the effects of training on children's ability to understand and memorize a text, evaluated in the Studying Test. Finally, with the sixth MANOVA, we aimed to analyze the effects of training on children's ability to solve mathematical problems, evaluated through the SPM. Univariate tests were performed where necessary.

The first MANOVA did not show a significant effect of the chess training on the approach to studying dimensions investigated by the QAS: Wilks/' Lambda $=0.928, \mathrm{~F}(7,77)=0.86, \mathrm{p}=0.54, \eta^{2}=0.072$. The children who participated in the chess training seem to approach the study in the same way as the control group.

The MANOVAs for the QS1 and QS2 questionnaires and for the index of strategic coherence were separately calculated: Wilks' Lambda $(\mathrm{QS} 1)=0.951, \mathrm{~F}(2,82)=2.09, \mathrm{p}=0.13, \eta^{2}=0.049$; Wilks' Lambda $(\mathrm{QS} 2)=0.994, \mathrm{~F}(2,82)=0.23, \mathrm{p}=0.79, \eta^{2}=0.006$; Wilks' Lambda (strategic coherence) $=0.979, \mathrm{~F}(2,82)$ $=0.89, \mathrm{p}=0.42, \eta^{2}=0.021$. The results show no significant difference between the experimental and control groups relative to the children's beliefs about the effectiveness of study strategies on the degree of their actual usage and on the degree of coherence with which they actually use study strategies they consider most effective.

From the fifth MANOVA, no effects of chess training on children's ability to understand and memorize a text (evaluated by the Studying Test) emerged: Wilks' Lambda $=0.954, \mathrm{~F}(3,81)=1.31, \mathrm{p}=0.28$, $\eta^{2}=0.046$.

The sixth MANOVA, which compared the two groups of children on their ability to solve mathematical problems (SPM test), instead highlighted a difference between the experimental and control groups: Wilks' Lambda $=0.801, \mathrm{~F}(6,78)=3.23, \mathrm{p}=0.01, \eta^{2}=0.199$. This last result has been examined in detail through a series of individual ANOVAs, one for each of the single dimensions investigated by the SPM battery.

The ANOVA findings indicate that chess training seems to primarily influence the ability to create a mental representation. This ability is measured by the SPM battery with a test in which the child must choose between a series of more or less abstract graphic representations (vignettes or diagrams) of the problem to be solved. 
Another dimension in which the children in the experimental group exhibited significantly better performance than those in the control group is that of categorization, which investigates children's ability to extend their knowledge on the solution to a given problem to other similar problems. Finally, the children in the chess group achieved better results in the self-assessment dimension, showing a greater ability to objectively assess their problem-solving performance.

Table 1. Analysis of Variance for the Control and Chess Training Groups' scores in SPM Battery Sub-tests $(\mathrm{n}=85)$

\begin{tabular}{|c|c|c|c|c|c|c|}
\hline & $\begin{array}{l}\text { Control } \\
\text { Group } \\
\text { Mean }\end{array}$ & $\begin{array}{l}\text { Control } \\
\text { Group } \\
\text { sd }\end{array}$ & $\begin{array}{l}\text { Chess } \\
\text { Training } \\
\text { Group } \\
\text { Mean }\end{array}$ & $\begin{array}{l}\text { Chess Training } \\
\text { Group } \\
\text { sd }\end{array}$ & $\mathrm{F}$ & p-value \\
\hline Problem comprehension & -0.09 & 1.66 & 0.01 & 1.83 & 0.07 & 0.79 \\
\hline Problem representation & -0.11 & 1.34 & 0.41 & 1.06 & 3.91 & $0.05^{*}$ \\
\hline Problem-solving planning & 0.25 & 1.05 & 0.26 & 0.83 & 0,00 & 0.98 \\
\hline Problem-solving execution & -0.59 & 0.98 & -0.60 & 1.28 & 0.00 & 0.99 \\
\hline Self-evaluation & -0.41 & 0.65 & -0.15 & 0.55 & 4.05 & $0.05^{*}$ \\
\hline
\end{tabular}

Mean score, sd, F, and $\mathrm{p}$-value $\left(* * * \mathrm{p}<.001,{ }^{* *} \mathrm{p}<.01,{ }^{*} \mathrm{p}<.05\right)$

\section{DISCUSSION AND CONCLUSION}

The aim of this work was to observe the effects of chess training on general meta-cognitive abilities and on skills closely related to school performance. Two groups of children were compared: an experimental group that participated in a chess training and a control group that participated in a sports program.

As for the general meta-cognitive abilities involved in learning, no significant differences emerged between the two groups in the different dimensions related to the QAS test assessing the general way in which they approach studying and some related sub-dimensions (e.g., motivation, strategic elaboration of the study material, ability to concentrate, and attitude toward schooling). No differences emerged between the two groups regarding children's beliefs about the effectiveness and the actual usage of study strategies. The studies that have explored the benefits of chess training on cognitive skills and school performance are scarce in the literature, and only one, to our knowledge, has specifically considered meta-cognitive abilities (Kazemi et al., 2012). In their study, the authors found that the effects of chess training were closely related to meta-cognitive abilities linked to math problem solving. The results of our study, in which we did not find an improvement in meta-cognitive ability after chess training, are not consistent with those of Kazemi et al. (2012). However, the skills considered in our study are quite different from the ones explored by Kazemi and colleagues: They tested meta-cognitive skills specifically involved in math problem solving, while we tested general meta-cognitive abilities applicable to any kind of subject of study. One potential explanation for our findings is that the dimensions tested in our study (e.g., motivation, attitude toward schooling, knowledge and usage of study strategies) are quite different from the skills acquired through chess practice, such as elaborating game plans. They require more than merely a simple transfer, but what Mestre (2005) defined as a far transfer, that is, a transfer between areas that are far from each other, and much more difficult to gain.

Although we have investigated only meta-cognitive abilities, our data seem to go in the direction of the studies that have investigated more general cognitive abilities, e.g., Scholz et al. (2008), who did not find an effect of training with chess on focused attention. A meta-analysis by Burgoyne et al. (2016) considered 19 studies that related cognitive abilities to chess skills and found a positive correlation between general cognitive abilities and chess practice that seemed, however, to be mediated by age and chess skill level. In particular, the younger and more inexperienced participants were, the greater the correlation with cognitive abilities. But it must to be noted that in the meta-analysis, the percentage of explained variance of cognitive abilities on chess performance is on average $6 \%$, a fairly low value. In the already cited meta-analysis done by Sala and Gobbet (2016), the authors underlined how in the works considered, the effect size is not large enough to strongly support the hypothesis that the improvement in cognitive abilities is due to the chess 
itself. Moreover, the authors highlighted that most studies considered did not consider the placebo effect: very often the control groups were not involved in other activities that could enhance their cognitive abilities. Therefore, it is difficult to determine whether the (already small) effect found was due strictly to playing chess or merely to being involved in a stimulating activity. The results of Sala and Gobbet's (2016) meta-analysis regarding cognitive abilities therefore seem to support the difficulty in the transfer of chess-related abilities to general cognition.

For the study trial in which participants were asked to understand and remember written text informational content, no significant difference was found between the chess and control groups. This is an expected result: The researchers who have investigated the relationship between chess and verbal skills have not found relationships between these two abilities, the authors; explanation lies in the fact that these two types of skills do not share common elements (see Sala \& Gobbet, 2016 for further discussion). However, it must be emphasized that in our work, we have considered the abilities of understanding and retaining information that have more in common with chess from the point of view of the underlying cognitive abilities than the simple reading skills investigated in other studies; despite this, however, we did not find significant effects.

Significant differences between the two groups of children have emerged, instead, in the SPM test score assessing the ability to solve mathematical problems, particularly in the dimensions of problem representation, problem categorization, and self-evaluation. Chess-training children are more capable of organizing information by creating a coherent representation of a problem and more able to categorize problems; they even demonstrate a greater capacity to extend their knowledge of a problem's structure to other similar problems to be able to solve them faster. The meta-analyses previously considered show that the domain of mathematics is the one that benefits most from chess training, leading most authors to think that these two areas involve common cognitive abilities. The literature results suggest that playing chess allows children to develop skills that can be de-contextualized, such as problem-solving skills and the ability of identifying quantitative relationships - abilities that can be transferred to the domain of mathematics. Our findings, demonstrating a difference between the experimental and control groups only in the math problem-solving domain, confirm this hypothesis.

Furthermore, our results showed that the chess players have a better capacity than the children in the control group to self-evaluate their school performance. This is in line with Aciego and Betancort's (2012) findings that chess practice improved not only cognitive skills but also the socio-emotional sphere, especially the ability to self-evaluate.

In sum, our findings confirm that chess practice can be useful for primary school children to enhance their mathematical problem-solving abilities and learning-related self-evaluation abilities even if chess practice could be of scarce use for improving meta-cognitive abilities applied to general school activity and for text comprehension, confirming literature data.

Our work has the merit of having used a sample taken from a primary school where the children were randomly assigned to an experimental group that played chess and to a control group. Often the works in this area use samples drawn from populations already selected a priori, such as children who are part of real chess clubs. In line with literature recommendations, the time extent of the training was 30 hours, and the control group was engaged in an alternative activity.

However, there are several limitations to note. The main limitation is that the participants did not undergo a pre-test to assess whether differences existed before the treatment, given that we had to limit the number of testing sessions to meet a school demand. To overcome this limitation, we used a large sample, randomly drawn from a school population and not pre-selected in a chess club. We selected classes in which the same teachers were regularly involved and asked the teachers, in advance, to evaluate whether the cognitive and academic levels of the children randomly assigned to the two groups were comparable. Nonetheless, we cannot exclude the possibility that one of the groups would start higher than the other in some of the dimensions considered. This is a serious limitation, even if the lack of a rigorous test-retest methodology is often observed in this type of work (see Sala \& Gobbet, 2016 for further discussion).

Our findings suggest that the topic of the transfer of skills gained through chess practice to the academic domain is worth further investigation. Future research should be undertaken using a pre-posttest experimental design and a longitudinal approach to investigate the effects of chess practice over time. 


\section{ACKNOWLEDGEMENT}

The authors would like to thank the principal of the school where the data collection took place. Special thanks go to the children, families, and teachers who participated in the study.

\section{REFERENCES}

Aciego, R., García, L., \& Betancort, M. (2012). The benefits of chess for the intellectual and social-emotional enrichment in schoolchildren. The Spanish journal of psychology, 15(2), 551-559.

Anderson, J. R. (1990). Cognitive psychology and its implication (3rd ed.). New York: Freeman.

Binev, S., Attard-Montalto, J., Deva, N., Mauro, M., \& Takkula, H. (2011). Declaration of the European Parliament, 0050/2011.

Burgoyne, A. P., Sala, G., Gobet, F., Macnamara, B. N., Campitelli, G., \& Hambrick, D. Z. (2016). The relationship between cognitive ability and chess skill: A comprehensive meta-analysis. Intelligence, 59, 72-83.

De Beni, R., Moè, A., \& Cornoldi, C. (2003). AMOS 8-15. Abilità e motivazione allo studio: Prove di valutazione e orientamento (Vol. 30). Edizioni Erickson.

Ericsson, K. A., \& Charness, N. (1994). Expert performance: Its structure and acquisition. American psychologist, 49(8), 725.

Gobet, F., \& Campitelli, G. (2006). Educational benefits of chess instruction: A critical review. In Chess and education: Selected essays from the Koltanowski conference (pp. 124-143). Chess Program at the University of Texas at Dallas Dallas, TX.

Kazemi, F., Yektayar, M., \& Abad, A. M. B. (2012). Investigation the impact of chess play on developing meta-cognitive ability and math problem-solving power of students at different levels of education. Procedia-Social and Behavioral Sciences, 32, 372-379.

Lucangeli, D., Tressoldi, P. E., \& Cendron, M. (1998). SPM-Test delle abilità di soluzione dei problemi matematici. Edizioni Erickson.

Mestre, J. P. (2005). Transfer of learning from a modern multidisciplinary perspective. Greenwich, CT: Information Age.

Perkins, D. N., \& Grotzer, T. A. (1997). Teaching intelligence. American psychologist, 52(10), 1125.

Sala, G., \& Gobet, F. (2016). Do the benefits of chess instruction transfer to academic and cognitive skills? A meta-analysis. Educational Research Review, 18, 46-57.

Sala, G., \& Gobet, F. (2017). Does chess instruction improve mathematical problem-solving ability? Two experimental studies with an active control group. Learning \& behavior, 45(4), 414-421.

Sala, G., Foley, J. P., \& Gobet, F. (2017). The effects of chess instruction on pupils' cognitive and academic skills: state of the art and theoretical challenges. Frontiers in psychology, 8, 238.

Scholz, M., Niesch, H., Steffen, O., Ernst, B., Loeffler, M., Witruk, E., et al. (2008). Impact of chess training on mathematics performance and concentration ability of children with learning disabilities. International Journal of Special Education, 23, 138e148.

Sternberg, R. J. (Ed.). (2000). Handbook of Intelligence. Cambridge: Cambridge, University Press.

Thorndike, E. L, \& Woodworth, R. S. (1901). The influence of improvement in one mental function upon the efficiency of other functions. Psychological Review, 9, 374-382. 